\title{
La nueva violencia urbana de América Latina
}

ROBERTO BRICEÑO-LEÓN*

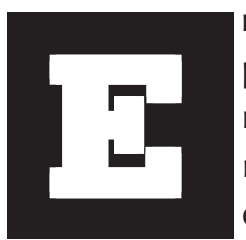

n América Latina no hay guerras al comenzar el Siglo XXI, pero las muertes por la violencia causan tantos hombres muertos, producen tantas mujeres viudas y dejan tantos niños huérfanos, como en los enfrentamientos armados que la televisión nos muestra que ocurren en otros lados del planeta. Y, en algunos casos, los daños son todavía mayores: las muertes que se podían contar después de un fin de semana normal en Caracas, Medellín o São Paulo, eran más que las que ocurrían en Kosovo durante la guerra; o son más que las víctimas de las infinitas retaliaciones del Medio Oriente. Y también, en otros casos, la paz que siguió a la guerra deja más muertos que los enfrentamientos entre el ejército y la guerrilla. La situación es novedosa y retadora para la sociología

La violencia es la primera causa de muerte entre las personas jóvenes y productivas que tienen entre 15 y 44 años de edad (WHO, 1999). En Colombia, entre 1985 y 1994, se duplicó el número total de viudas, mientras que los viudos apenas aumentaron levemente, pues son viudas de la violencia y los hombres son sus víctimas principales. Y los huérfanos, que para 1985, calculaban en 43 mil niños, se incrementaron a 73 mil huérfanos en 1994. (Rubio, 2000, p. 124-125). En El Salvador, Centroamérica, el gobierno y la guerrilla con mediación internacional, pusieron fin a una

\footnotetext{
*Sociólogo, PhD, Director del Laboratorio de Ciencias Sociales (LACSO) y Profesor Titular de Sociología de la Universidad Central de Venezuela. Es miembro del Comité Ejecutivo de la International Sociologial Association (ISA) y Coordinador del Grupo de Violencia y Sociedad del Consejo Latinoamericano de Ciencias Sociales (CLACSO).
} 
guerra interna muy cruenta, pero la tasa de homicidios se incrementó de 72 a 139 homicidios por cada cien mil habitantes entre 1990 y 1995 (Cruz, Trigueros y González, 2000). En América Latina encontramos más muertes en la calma de la paz que en las tormentas de la guerra.

La violencia no ha sido ajena a los procesos de cotidianidad o transformación social de América Latina: violenta fue la conquista, violento el esclavismo, violenta la independencia, violentos los procesos de apropiación de las tierras y de expropiación de los excedentes. Pero en la actualidad hablamos de un proceso distinto, singular, y que se refiere a la violencia delincuencial y urbana. Ciertamente la violencia política ha estado presente en la región, la represión militar de los gobiernos dictatoriales del Cono Sur o Centroamérica; las luchas guerrilleras recientes en Perú, Colombia o México; las tradicionales acciones de los "coroneles", los señores de la tierra, del nordeste de Brasil o las acciones de los paramilitares en Urabá, Colombia, son una muestra fehaciente de ello. Pero, cuando uno observa las tasas de homicidios para todos estos países hasta comienzos de los años ochenta y lo que después ocurre, la situación no es comparable, no tiene la gravedad que después muestra, justamente cuando desaparecen las dictaduras, amainan las guerrillas y se decreta la paz y la democracia.

Se trata entonces de una violencia distinta. Una violencia que podemos calificar de social, por expresar conflictos sociales y económicos; pero no de política, pues no tiene una vocación de poder. Una violencia que no tiene su campo privilegiado de acción en las zonas rurales, sino en las ciudades y, sobretodo, en las zonas pobres, segregadas y excluidas de las grandes ciudades, donde a veces como en los pistoleros de Brasil se trasladan prácticas rurales a la vida urbana (Barreira, 1998). Y todo esto ocurre a partir de los años ochenta, pues es desde mediados de la "década perdida" cuando, en todos los países de los cuales se dispone información confiable, se incrementó la violencia. Los crímenes violentos aumentan tanto en aquellos países con muy bajas tasas de homicidios - como Costa Rica o Argentina - hasta los 
otros que ya las tenían muy altas - como Colombia o El Salvador.

Comprender esta realidad implica pasearse por diversos aspectos y dimensiones del problema y de las transformaciones que en la situación social se han venido dando en América Latina.

\section{La pobreza y el empobrecimiento}

Un rasgo muy significativo de la nueva violencia urbana es que ella ocurre primordialmente entre los pobres de las grandes ciudades. La clase media y los sectores adinerados ven los pobres como una amenaza, y se sienten a sí mismos como las víctimas de las agresiones y delitos. Pero esto es sólo parcialmente cierto. Es la clase media, por supuesto, que sufre la delincuencia, pero, quienes verdaderamente padecen la violencia y, en particular, la violencia más intensa o letal, son los pobres mismos quienes son víctimas y victimarios en este proceso (Briceño-León, Camardiel y Avila, 1998). Es una violencia de pobres contra pobres.

Pudiera tenerse la tentación de derivar, a partir de esta cruda realidad, la conclusión que la pobreza es la causa de la violencia. Sin embargo, no existe una correlación tan clara y abierta entre estas dos situaciones, pues los países más pobres de América Latina como serían Haití, Bolivia o Perú, no aparecen entre aquellos que tienen mayores tasas de homicidios. Y lo mismo ocurre a lo interno de los países, la mayor violencia de Brasil no se encuentra en los pobres y sedientos Estados del nordeste, sino en los ricos y cosmopolitas estados de São Paulo y Rio de Janeiro (Zaluar, Noroña y Alburquerque, 1994). En Venezuela, los homicidios ocurren en el Área Metropolitana de Caracas y en los ricos estados de Carabobo y Aragua y no en las pobres entidades, con mayores necesidades básicas insatisfechas, como Apure, Trujillo o Sucre (Briceño-León y Pérez Perdomo, 2000).

Ante esta situación hemos planteado una hipótesis alternativa: es el empobrecimiento y la desigualdad, y no la pobreza, lo que origina la violencia urbana que estamos presenciando. Es el empobrecimiento que 
ha hecho que para 1998, en 13 de 18 países de la región, el salario mínimo fuera inferior al de 1980 y que el número total de pobres superase los 220 millones de personas (CEPAL, 2000).

\section{La exclusión escolar y laboral}

Los estudios recientes del Banco Mundial toman como medida de la pobreza, el umbral de un dólar por persona por día (para lo cual se considera el dólar con paridad de poder comprar a precios de 1985), pues bien, según estos cálculos el 24\% de la población de América Latina y el Caribe vive con menos de un dólar por día, es decir, uno de cada cuatro latinoamericanos. Y en algunos países, como Guatemala, más de la mitad de la población se encuentra en esa situación (World Bank, 2001). Esto genera una situación creciente de exclusión entre la población, pero esa exclusión se observa más claramente en el trabajo y en la educación.

Según la CEPAL, el desempleo en la región pasó de 5,7\% en 1990 a 9,5\% en 1999, pero lo que llama la atención no es tan sólo el incremento de los desocupados, sino la particularidad de los nuevos trabajos, pues de cada diez empleos que se crearon en la región entre 1990 y 1997, siete (6,9 exactamente) se originaron en el sector informal (CEPAL, 1999). Es decir, ocurre una doble exclusión laboral, pues hay menos empleos y aquellos que surgen tienen un carácter tan precario como su condición de informalidad lo sugiere.

Desde el punto de vista educativo la situación no es mejor. Se calcula que el 30\% de los niños no había completado la educación primaria al cumplir los 14 años de edad. Y al tener esa edad ya se ven obligados a trabajar y deben salir al precario mercado laboral a buscar algo de dinero, pues sus padres - los que existan y los acompañen -, ya no pueden continuar financiando sus básicas o crecientes necesidades de consumo, pero, también, porque deben contribuir con el mantenimiento de la familia, así sea no representando una carga y permitiendo que mayores recursos puedan 
Sociologias, Porto Alegre, ano 4, no 8, jul/dez 2002, p. 34-51

distribuirse entre los hermanos menores.

La educación funciona relativamente bien como puerta de entrada, pero muy mal como puerta de salida. Es decir, en el inicio de los estudios se logran incorporar un porcentaje bastante alto de los niños, sobre todo en las zonas urbanas, pero la prosecución de los estudios es muy baja. Si tomamos como referencia los 9 años que se consideran debe al menos estudiar cualquier persona, y así se encuentra establecido en las leyes de muchos países como educación obligatoria, los resultados son muy poco halagadores. Por ejemplo, se calcula que de cada 100 niños que ingresan al primer grado de la escuela en Bolivia, Brasil, Colombia o Perú, sólo 15 llegan al noveno grado de estudios. Pero la situación es aún peor en otros países como Guatemala, Haití o República Dominicana, donde de los mismos 100 alumnos sólo 6 alcanzan los nueve años de estudio (BID, 1998).

En Caracas, Venezuela, el 27\% de los jóvenes hombres entre 15 y 18 años de edad, ni trabaja ni estudia (Briceño-León, 1997), ¿Qué puede uno esperar de un adolescente ocioso en su casa, o, peor aún, en la calle, expuesto a todos los peligros y las tentaciones posibles?

La exclusión laboral y educativa son factores de gran relevancia para el crecimiento de la violencia en las ciudades latinoamericanas. Ciertamente el proceso educativo formal no logra apartar del todo a los jóvenes de la violencia. Pero la no-educación, es decir, el proceso de expulsión de las escuelas que sufren los jóvenes hace la situación aun peor, pues éstos no logran insertarse en el mercado laboral y quedan sin vínculos sociales formales que les puedan proporcionar un sentido de futuro. Este fenómeno no ocurre así en las zonas rurales, pues allí al salir de la escuela pueden tener un trabajo, una ocupación y un sentido del futuro que aunque modesto le permite al adolescente organizar la cotidianidad del presente. Por ello la violencia prospera más entre quienes nacieron en la ciudad y alimentaron sus sueños con el progreso urbano. 


\section{La segunda generación urbana y el quiebre de las expectativas}

Uno de los rasgos significativos de la violencia urbana es que, al contrario de lo que podía esperarse, de acuerdo a las tesis de la sociología de la modernización, no ocurrió cuando el grupo de inmigrantes llegaba a las ciudades provenientes del campo y había perdido sus controles sociales tradicionales. La violencia ocurre en la segunda o tercera generación urbana, en individuos que nacieron en las ciudades y que habían perdido todo vínculo y memoria con su pasado rural. La hipótesis que hemos propuesto para explicar esta situación radica en la insatisfacción de las expectativas que se genera en las generaciones pobres que han nacido en las ciudades.

Dos componentes tiene esta situación. Por un lado está el proceso de logro de las aspiraciones que tuvo la primera y, en algunos países hasta la segunda generación. Para las familias que venían del campo, la ciudad representaba un conjunto de beneficios importantes que no podían obtener en sus lugares de origen: en la ciudad podían tener un hospital cercano, la escuela para los hijos, agua potable en la casa o cerca de ella, la electricidad y con ello una refrigeradora y un televisor; en fin, algunas circunstancias que implicaban, aún en su precariedad, un cambio importante en la calidad de vida. Es de destacar que en América Latina este proceso migratorio que en la literatura de la época se denominó el "éxodo rural-urbano", coincidió con una etapa importante de expansión del capitalismo y mejoría de las condiciones sociales de la economía mundial, es decir, en el período posterior a la Segunda Guerra Mundial. Aunque en algunos países el aceleramiento del proceso de urbanización ocurrió desde antes y fue sostenido por las exportaciones previas y durante los años de la guerra, en su conjunto podemos establecer que en los años cincuenta se acrecentó un movimiento migratorio importante que creó ese nuevo fenómeno urbano que constituyen las favelas, villas miserias, comunas o pueblos jóvenes y que la sociología llamó de maneras tan diversas como marginalidad, asentamientos urbanos no planificados o sobrepoblación relativa. 
Pero los individuos que nacen en la ciudad no encuentran nada novedoso en la electricidad, la televisión, los hospitales o las escuelas. Siempre estuvieron allí. En cambio, sus aspiraciones son otras. Y es aquí donde ingresa el segundo aspecto de la sociedad contemporánea que deseamos destacar: la homogeneización e inflación de las expectativas. La presencia permanente de los medios de comunicación, y sobre todo de la televisión, coloca a los individuos de los más distintos niveles sociales y capacidad adquisitiva en contacto con un conjunto de bienes, servicios y estilos de vida que muchos de ellos antes no podían conocer o imaginarse. De alguna manera, los medios de comunicación y la publicidad han democratizado el acceso a la información acerca de los productos y servicios que existen en la oferta del mercado, y con ello han hecho que se incrementen las expectativas en la población de una manera importante. Es decir, todos los jóvenes pueden desear el mismo tipo de camisa, la misma marca de zapatos y el mismo modelo de automóvil, pero no todos y por igual tienen similares capacidades de comprarlos. Todos tienen la libertad de comprarlo, pero no la capacidad de hacerlo, pues una gran cantidad está desempleada o gana sueldos mensuales que son inferiores al costo de un par de zapatos deportivos de la marca de moda.

El proceso de homogeneización e inflación de las expectativas en la segunda o tercera generación urbana ocurre al mismo tiempo que se detienen el crecimiento económico y las posibilidades de mejoría social y se produce un abismo entre lo que se aspira como calidad de vida y las posibilidades reales de alcanzarlo. Este choque, esta disonancia que se le crea al individuo entre sus expectativas y la incapacidad de satisfacerlas por los medios prescritos por la sociedad y la ley, son un propiciador de la violencia, al incentivar el delito como un medio de obtener por la fuerza lo que no es posible de lograr por las vías formales. 


\section{La disponibilidad de armas de fuego y la construcción de la masculinidad}

Según cifras de la Organización Mundial de la Salud, el 63\% de los homicidios que ocurren en el mundo son ocasionados por armas de fuego, pero esta cifra es muy superior en América Latina, donde supera al $80 \%$ y en algunos países como Venezuela es mayor del 90\% (WHO, 1999; Londoño y Guerrero, 2000).

La difusión de armas de fuego ligeras entre la población de América Latina ha crecido de una manera impresionante en los últimos veinte años. En algunos países existe un libre mercado de armas de fuego, en otros hay mayores restricciones, pero muchas de las armas que estuvieron en manos de la guerrilla pasaron a usuarios privados y a la delincuencia común. Los comerciantes de la droga se encargaron también de entregar armas a sus distribuidores como parte de los pagos y como un medio de defensa de sus territorios. Y los ciudadanos honestos también decidieron armarse para defender sus propiedades y sus familias, y si bien las cifras reales son muy difíciles de obtener, una encuesta del año 1997 mostraba que el 23\% de los habitantes de Cali y de San José de Costa Rica, o el 28\% de los habitantes de Santiago de Chile, tendrían un arma de fuego si pudieran (Activa, 1998).

Los mercados de las armas son muy complejos y se vinculan con un comercio y una industria que tienen muchas ramificaciones en la sociedad y los negocios. Países de Europa, muy estrictos con el porte de armas a sus ciudadanos, se niegan a imponer control a las exportaciones de pistolas y revólveres de sus industrias para otros países; se supone que las venden a empresas "serias", pero luego pasan al mercado negro de armas en el mundo y, en su fases finales, involucran a las policías o ejércitos locales quienes se convierten en los principales contrabandistas de armas. Al final, todo el mundo que quiere puede adquirir un arma para delinquir o defenderse, $y$, quien no puede comprarla, tal y como ocurre en Caracas, le es posible 
Sociologias, Porto Alegre, ano 4, no 8, jul/dez 2002, p. 34-51

alquilarla por un fin de semana.

Lo que tiene de singular la violencia de América Latina, como también de los Estados Unidos de América y del mundo contemporáneo, no es la existencia de más delitos ni de mayores conflictos interpersonales, sino la letalidad de dicha violencia. Es decir, no se trata de que la gente pelea más, sino que se mata más (Zimring \& Hawkins, 1997). Y la letalidad esta intrínsecamente relacionada con la posesión de armas de fuego que son capaces de asesinar mucho más fácilmente que las armas blancas.

Pero el arma tiene además de su función utilitaria una función simbólica importante. El arma representa la masculinidad, el valor y la capacidad de defenderse y demostrar su hombría y su coraje entre los jóvenes. Es muy relevante señalar que cerca del 90\% de las víctimas de homicidios son hombres. Son los hombres quienes en la construcción cultural de su masculinidad le corresponde el rol de osados y valientes y donde la conducta de evitación del conflicto es identificada claramente como un rasgo femenino que ningún hombre debiera imitar si desea seguir considerado como tal entre sus pares (Márquez, 1999; Zubillaga y Briceño-León, 2001).

Buena parte de la violencia urbana debemos atribuirla a estas dimensiones culturales de la masculinidad. Sobre todo esto se vuelve aun más marcado entre los adolescentes quienes se encuentran en una fase de definición de su identidad y que, por lo tanto, son más vulnerables a este tipo de valores, pues deben sistemáticamente demostrar que no son más niños, sino hombres, aun a costa del riesgo de morir o matar por cualquier trivialidad.

\section{El miedo a ser víctima y los costos económicos de la violencia}

El incremento de las tasas de homicidios y los delitos contra la propiedad han creado una sensación de temor generalizada entre la población urbana de América Latina. Si hay algo común entre los habitantes de las distintas 
metrópolis es la sensación de miedo a ser víctima de la violencia. El estudio Latinobarómetro mostró que en promedio el 30\% de las familias de la región habían sufrido un robo o un asalto en los doce meses anteriores a la encuesta, es decir, una de cada tres familias. Los resultados variaban entre algunos países que como Guatemala superaban el 50\%; o estaban muy cerca de la mitad, como México y El Salvador). Otros países como Argentina (34,2\%) Bolivia (32,8\%), Costa Rica $(32,7 \%)$ o Chile $(32,0 \%)$ estaban cercanos a la media. Solamente Panamá $(25,1 \%)$ y Uruguay $(21,4 \%)$ mostraron el menor porcentaje de víctimización y aún así se trataba de una de cada cuatro o cinco familias (Londoño y Guerrero, 2000).

Pero el miedo es aun mayor que la probabilidad real de ser víctima, pues ese $30 \%$ que ha sufrido de robos o asaltos, tiene vecinos y amigos a quienes le cuentan lo que les aconteció, y quienes empiezan a sentirse en forma vicaria víctimas reales o potenciales, y es así que el miedo se generaliza de una manera importante, limitando la libertad de las personas por las restricciones que a sí mismos se imponen los individuos (Zubillaga y Cisneros, 2001). En el estudio ACTIVA (1998) se encontró que el 24\% de los encuestados en Río de Janeiro, el 26\% en Santiago de Chile y el el 46\% de Cali, habían restringido sus salidas durante la noche por temor a ser víctimas de la violencia. En Caracas, cerca del 33\% de las personas habían restringido sus horarios nocturnos de estudio o trabajo por las mismas razones. Pero ese mismo temor invade a los habitantes de ciudades mucho más seguras como Buenos Aires o Montevideo, o a los habitantes de otros centros urbanos de menor magnitud en población y violencia, pero que, por efecto de los medios de comunicación, viven vicariamente los sucesos de otros lugares y tienden a sentir un temor muy superior al que objetivamente correspondería con las probabilidades de ser víctima en el lugar donde habitan.

Esta sensación de miedo tiene un impacto económico muy grande en la sociedad, y agiganta los ya importante daños que la misma violencia 
y el delito tienen sobre el patrimonio de las sociedades. Pues, a los ya relevantes costos directos que sufren por pérdidas en la salud de la población y por pérdidas materiales, es necesario sumar los gastos que tienen que hacer las familias y las empresas para protegerse y los costos indirectos que ocasionan como resultado de la inhibición de la actividad económica, por las personas que no salen a comprar o que no trabajan horas extras.

Los costos económicos directos de la violencia, en Colombia, representan el 11,4\% del Producto Interno Bruto (PIB) y el 6,9\%, en El Salvador (Rubio, 2000; Cruz, Romano, González y Sistí, 2000). De los estudios de caso que se han realizado, estos son los dos países donde el impacto económico es mayor, pero aunque de menores magnitudes, también son importantes dichos costos directos en Venezuela, donde es el 6,9\% (Navarro, 1999), en México es del 4,9\% (Lozano et al., 2000) y en Brasil y Perú del $3,3 \%$ y $2,9 \%$ respectivamente (Couttolene, Cano, Carneiro y Phebo, 2000; Eyzaguirre, 2000). La cifra menor de todos estos países la tiene Perú, pero aun así es superior a lo que por años se ha solicitado que como porcentaje del PIB debe dedicarse a la Ciencia y la Tecnología para lograr su desarrollo. Pero estas cifras ascienden de manera notable, duplicándose o triplicándose en algunos países cuando se incluyen los costos indirectos y las transferencias, Ilegando a representar el 24,9\% del PIB en El Salvador, el 24\% en Colombia, el 12,3\% en México, el 11,8\% en Venezuela, el 10,5\% en Brasil y el 5,1\% en Perú. Los daños y transferencia de recursos que se dan en la región como resultado de la violencia representan, según cálculos del Banco Interamericano de Desarrollo, el 14,2\% del Producto Interno Bruto, lo cual es el equivalente a 168.000 millones de dólares (Londoño y Guerrero, 2000). La violencia tiene entonces, además de los costos de dolor y sufrimiento humano, un componente de malestar psicológico derivado del miedo que inspira y un impacto económico, por las víctimas y costos reales, pero, también, por los gastos y pérdidas que su prevención y miedo obligan. 


\section{Violencia ciudadana y violencia policial}

Tanto la victimización real como el miedo han impulsado una respuesta violenta por parte de la población. Respuesta violenta que tiene una dimensión actitudinal por medio de la cual se está propenso a aceptar las acciones violentas de la policía, a exigir un incremento en la severidad de las penas, a apoyar la pena de muerte, a armarse y estar dispuesto a matar para defender su propiedad y su familia. Más del $60 \%$ de los pobladores de Santiago de Chile, San José de Costa Rica, San Salvador, Río de Janeiro y Caracas, considera que se tiene derecho a matar para defender a su familia. Pero estas respuestas pudieran justificarse a los ojos de algunas personas por ser defensivas, pero el nivel más bajo de aprobación para el apoyo a las "limpiezas sociales", es decir, el asesinato de delincuentes y criminales por parte de grupos parapoliciales, es del 36\%, pero hay ciudades donde puede duplicarse ese porcentaje (Briceño-León, Camardiel y Avila, 1999).

Los linchamientos se han venido convirtiendo en una respuesta relativamente familiar entre los pobladores de las zonas pobres de América Latina. Quizá el número es todavía bajo, pero la posibilidad se contempla con mucha más frecuencia y normalidad entre las personas que no encuentran un apoyo en la policía para defenderse de los agresores, no hay castigo a los delincuentes por parte del sistema judicial. Ante esa situación de indefensión, que es vivida como cercana a la anomia en algunas comunidades, el linchamiento se convierte en una alternativa (Vila, 2001).

De igual modo se observa como se ha incrementado la violencia de la policía en sus actuaciones. En muchos casos, esta violencia ha sido propiciada de manera formal por las autoridades, como fue el caso de los premios de "bravura" que la policía de Río de Janeiro daba a los oficiales que se mostraban agresivos con los delincuentes (Soares, 2000). Otras veces, como extensión de esas órdenes, por los códigos de honor y solidaridad no escritos, o por simple exceso, la policía empezó a usar la fuerza letal de 
manera excesiva, arrojando, como se pudo conocer muy bien en un estudio en Brasil, más heridos que muertos y los muertos con muchos más impactos de balas que los que habían recibido los delincuentes en otras partes del mundo por la policía (Cano, 1997).

Pero llama la atención que estos hechos en lugar de tener repudio por parte de la población, ya que son claramente ilegales, pueden contar con su apoyo y su simpatía. En una encuesta en Caracas, el 34\% de la población aprobó la idea que la policía tiene derecho a matar a los delincuentes, y un viceministro de seguridad se pudo dar el lujo de declarar públicamente que la policía de Venezuela había eliminado a más de dos mil "predelicuentes", y todo esto sucedió en un país que no contempla la pena de muerte entre sus castigos. La violencia policial tiene un componente importante en el apoyo que los ciudadanos le dan a las acciones extrajudiciales y esto tiene una significación social muy notable, pues ya no se trata que los cuerpos policiales cometan excesos o actúen al margen de la ley por iniciativa propia, sea como vengadores solitarios a nombre de la justicia o sea como delincuentes escondidos detrás del uniforme. Se trata de una acción extrajudicial que cuenta con la simpatía de la población que se siente amenazada y no cree en la capacidad punitiva de las instituciones de justicia penal (Briceño-León, Piquet Carneiro y Cruz, 1999).

\section{El Sistema Penal y la Justicia}

La nueva violencia urbana plantea retos importantes al sistema de justicia penal por la singularidad del fenómeno que estamos describiendo. Ciertamente, como escribió Durkheim (1978), el crimen es normal en las sociedades, es decir, tan normal es que las normas se cumplan, como esperar que alguien las infrinja. El problema con el sistema penal es que el control social que se podía esperar que cumpliera, sólo puede ser eficiente si los infractores son unos pocos, pero se vuelve completamente inoperante cuando 
adquiere las magnitudes que hemos visto tiene la nueva violencia urbana.

El sistema penal enfrenta entonces un doble tipo de dificultades, por un lado no tiene la capacidad de hacer cumplir sus propios cometidos, la cantidad y variedad de delitos e infractores lo ha hecho inoperante en muchos países. La pena ha perdido la función disuasiva que se presume debe tener, y esto sucede tanto porque la probabilidad real de aplicarse es muy baja, como porque la fuerza simbólica que debe tener la ley se ha perdido o nunca se consolidó entre grupos importantes de la población. Si en el mundo se sabe que el sistema penal castiga apenas a una pequeña parte de los criminales, en las nuevas condiciones de la criminalidad esto se ha vuelto mucho mayor. En Colombia, durante los años sesenta, el 35\% de los homicidios cometidos llegaban a juicio; en los años noventa este porcentaje se había reducido al 6\% (Rubio, 2000); mientras en Cali se calcula que ni siquiera el 5\% de los homicidios llegan a juicio (Guerrero, 1996). Proceso Judicial que de ningún modo quiere decir que los culpables hayan sido condenados, pues en ese caso el porcentaje sería aún menor. Todo esto lleva a que el sentimiento de impunidad que experimenta la población es muy grande, pues los castigos son pocos y tardíos.

Pero, por otro lado, aun suponiendo que pudiera ser eficiente juzgando y condenando a los delincuentes, quedan las preguntas fuertes de la función social que ello cumpliría. Es decir, ¿puede el sistema penal y la prisión contribuir a disminuir la violencia? No pareciera que esto es claro, ya que las cárceles han llegado a ser los lugares más peligrosos cuando se presume deben ser los lugares más seguros: en Venezuela, la tasa de homicidios de las prisiones es muy superior a la del resto de la sociedad (Pérez Perdomo y Rosales, 1999) y pareciera que existe un amplio consenso en que las cárceles se convierten más en una fábrica que un correccional de violentos.

Las dudas teóricas sobre el sistema de justicia penal y las ineficiencias prácticas de su aplicabilidad, unas provenientes de la criminología crítica y otras de la sociología, obligan a repensar lo que puede hacer la sociedad con la nueva violencia urbana. Las viejas fórmulas de las cuales se disponía 
pareciera que no se ajustan más a las nuevas realidades, y que es necesario inventar caminos de mediación entre posturas muy hermosas, desde la perspectiva libertaria y emancipatoria hasta realidades y urgencias prácticas a las cuales debe dar respuesta un sistema policial y penal. Una justicia y una policía democrática pueden no ser utopías tan lejanas, pero mientras esto llega, la violencia se incrementa y se debilitan más las instituciones de justicia: en las seis ciudades del estudio Activa de la OPS, uno de cada tres entrevistados consideraba que los juzgados de su país eran malos o muy malos.

\section{Un reto para la sociología}

Los procesos sociales anteriormente descritos conforman una parte importante del escenario de violencia que enlútese a la región. No son todas las dimensiones que pudieran estudiarse, pero sí las más comunes a los distintos países y las que tienen mayor relevancia para la sociología. La nueva violencia urbana de América Latina es un reto para los estudios sociológicos, pues obligan a redefinir muchos de los conceptos y de las teorías que hemos heredado sobre la organización social y el comportamiento de los individuos. Esta necesidad de desarrollar teorías apropiadas para comprender la violencia se vuelve ineludible, tanto por la urgencia de responder a la singularidad que ha tenido América Latina como sociedad, así como por la necesidad de dar cuenta del proceso de globalización que ocurre en el mundo y en la región. Es decir, tanto la singularidad cultural y social previa de la violencia autóctona, como los nuevos factores que afectan la mundialización de la violencia (Tavares dos Santos, 2002) se combinan para generar este fenómeno singular que es la nueva violencia urbana de América latina. La gente mata y muere por esa singular combinación de los factores tradicionales y globalizados: por la cultura del honor rural y los zapatos de moda del jugador de básquetbol norteamericano; por la miseria de siempre y por la nueva, la que surge del empobrecimiento que produce la nueva economía, capaz de reportar crecimiento económico e incremento del desempleo al mismo 
tiempo. La nueva violencia se corresponde con la nueva sociedad que ha surgido en América Latina.

\section{Referencias}

ACTIVA. Estudio Multicéntrico sobre normas y actitudes hacia la violencia. Washington: OPS, 1998.

BARREIRA C. Crimes por encomenda: violência e pistolagem no cenário brasileiro. Rio de Janeiro: Relume-Dumará, 1998.

BID. Informe de Progreso Económico y Social. Washington: BID, 1998.

BRICEÑO-LEÓN, R; CARNEIRO, Piquet L. Y CRUZ, J.M. O apoio dos cidadãos à acão extrajudiciál da polícia no Brasil, em El Salvador e na Venezuela. En: D. Chaves Pandolfi, J. Murilo de Carvalho,'L. Piquet Carneiro e M. Grynzpan (Orgs.). Cidadania, justiça e violência. Rio de Janeiro: Editora da Fundaçáo Getülio Vargas, 1999, p. 117-127.

BRICEÑO-LEÓN, R.; A.Camardiel y O. Avila. Violencia y Actitudes de Apoyo a la Violencia en Caracás. Fermentum, Año 9, n' 26, Septiembre-Diciembre, 1999, p. 325-35.

BRICEÑO-LEÓN R. Buscando explicaciones a la violencia, en Espacio Abierto, Vol. 6, n' 1. p. 45-69.

BRICEÑO-LEÓN, R. y PERDOMO, R. Pérez. Violencia en Venezuela: un fenómeno capital. En: J. L.' Londoño, A. Gaviria y R. Guerrero (Eds.). Asalto al desarrollo; violencia en América Latina. Washington: Banco Interamericano del Desarrollo, 2000, p. 263-288.

BRICEÑO-LEÓN, R.; CAMARDIEL, A. y AVILA, O. ¿Quiénes son las víctimas de la violencia en Carácaś? Un análisis social del riésgo de la violencia no-fatal. Tribuna del Investigador, Vol 5, n⿳1, 1998, p. 5-P9.

CANO, I. The use of lethal force by police in Río de Janeiro. Rio de Janeiro! ISER, 1997.

CEPAL. La brecha de la equidad. Santiago de Chile: CEPAL, 1999.

CEPAL. Equidad, desarrollo y ciudadanía. Santiago de Chile: CEPAL, 2000. COUTTOLENE, B.; CANO, I; CARNEIRO, L. P. y PHEBO, L. Violencia y policía en
Rio de Janeiro Eñ: J. L. Londoño A. Gaviria y R. Guerrero (Eds.). Asalto al Desarrollo: Violencia en América Latina. Washington: Banco Interamericano del Desarrollo, 2000, p. 59-102.

CRUZ, J. M., GONZÁLEZ, A, ROMANO, E. y SISTí, E. De la Guerra al Delito: evolución déla violencia en El'salvador. En!: J. L. Londóño, A. Gaviria y R. Guerrero (Eds.). Asalto al desarrollo: violencia en América Latina. Washington: Banco Interamericano del Desarrollo, 2000, p. 173-203. 
CRUZ, J. M., TRIGUEROS ARGÜELLO, A. y GONZÁLEZ, F. El crimen violento en EI'Salvádor: Factores sociales y económicos asociados. San Salvador: IUDOP, 2000.

DURKHEIM E. Les règles de méthode sociologique. Paris: Press Universitaires de France, 1978.

EYZAGUIERRE, H. Ataque a la violencia en Perú: Reforma Penal y Policíaca. En: J. L. Londoño, A. Gaviria y R. Guerrero (Eds.). Asalto al desarrollo: violencia en América Latina. Washington: Banco Interamericano del Desarrollo, 2000, p. 233-260.

GUERRERO, R. Políticas de Combate ao Narcotráfico na América Latina. En: Lei e Liberdadé, v. 15, n. 47, 1996, p.11-13.

LONDOÑO J. L y GUERRERO, R. Violencia en América Latina: Epidemiología y Costos. In: J. 'L. Londoño A. Gaviria y R. Guerrero (Eds.). Asalto al Desarrollo: Violencia en América Latina. Washington: Banco Interamericano del Desarrollo, p. 11-57.

LOZANO, R.; HIJAR M.; ZURITA, B.; HERNÁNDEZ, P; AVILA, L.; BRAVO, M. L.; RAMIREZ, T.'j.; CARRILL'O, C.; AYALA, C.; LOPEZ, E'. C'apital Lésiónada: Violenciá en Ciudad de México. En:' J. L'. Londoño,'A. Gaviria y R. Guerrero (Eds.). Asalto al Desarrollo: violencia en América Latina. Washington: Banco Interamericano del Desarrollo, 2000, p. 205-232.

MARQUEZ, P. The street is my home. Stanford: Stanford University Press, 1999.

NAVARRO, J.C. Los costos del delito violento en Venezuela, Fermentum 9 (26), 1999, p. 383-396.

PÉREZ PERDOMO, R y ROSALES, E. La violencia en el espacio carcelario. Revista de Derecho Pénal (España), v. 2, n. 3, 1999, p. 293-313.

RUBIO, M. El desbordamiento de la violencia en Colombia. In: J. L. Londoño, A. Gaviria y R. Guerrero (Eds.). Asalto al Desarrollo: Violencia en América Latina. Washington: Banco Interamericano del Desarrollo, 2000, p. 103-171.

SOARES, L. E. Meu casaco de general. São Paulo: Companhia das Letras, 2000.

VILA, C. (In)justicia por mano propia:linchamientos en México contemporáneo. Revista Mexicana de Sociología v. LXIII, n. 1, p. 131-160.

WHO. Injury, a leading cause of the global burden of disease. Geneva, 1999.

WHO, World Bank beyond economic growth. Meeting the challenges of global development. Washington: World Bank, 2001.

TAVARES DOS SANTOS, J.V. The worldization of violence and injustice. Current Sociology, v. 50, n. 1, 2002, p.123-134.

ZALUAR, A. NOROÑA, J. y ALBUQUERQUE, C. Violência: pobreza ou grandeza instituciónal. Cadernos de Saúde Pública, 10 (supl.1), 1994, p. 213-217. 
ZIMRING, F. E. \& HAWKINS, G. Crime is not the Problem. Lethal violence in America. New York: Oxford University Press, 1997.

ZUBILLAGA, V. y CISNEROS, A. El temor en Caracas: relatos en barrios y urbanizaciones. Revista Mexícana de Sociología, v. LXXIII, n. 1, p.161-176.

ZUBILLAGA, V. y BRICEṆ̃̃-LEÓN, R. Exclysión, Masculinidad y respeto: Algunas Claves para entender la violencia éntre adolescentes en barrios. Nueva Sociedad, 173, Mayo-Junio, 2001, p 34-78.

\section{Resumen}

El artículo procura mostrar que la situación de violencia que se presenta a inicios del Siglo XXI en América Latina es un fenómeno novedoso desde el punto de vista sociológico, tanto por el crecimiento en sus magnitudes, como por lo singular de sus características. Se trata, argumenta el autor, de una violencia que no tiene orígenes o propósitos políticos, sino sociales y está vinculada al proceso de empobrecimiento que ha tenido la región a partir de los años ochenta y que ha creado unas condiciones de exclusión educativa y laboral para una gran parte de la población. Esta nueva violencia afecta fundamentalmente a los jóvenes de la segunda generación urbana quienes están expuestos a unas altas expectativas de consumo que no pueden satisfacer por los medios proscritos por la sociedad, y quienes ven en la violencia y el uso de las armas de fuego un medio para construir su identidad como hombres y lograr medios económicos para satisfacer sus aspiraciones. Este proceso de violencia delincuencial se ve acompañado de un incremento en la violencia policial, todo lo cual tiene grandes costos psicológicos y económicos para la población, costos y daños que se ven agravados por la desconfianza e ineficencia del sistema de justicia penal existente en América Latina.

Palabras-claves: violencia, criminalidad, situación social, sistema penal, América Latina. 